\title{
Dicer affects cisplatin-mediated apoptosis in epithelial ovarian cancer cells
}

\author{
XI WANG $^{1 *}$, HUI CHEN $^{1 *}$, YIPING WEN ${ }^{1}$, XIAOXIN YANG ${ }^{2}$, QING HAN $^{1}$, \\ PING JIANG $^{3}$, ZAIJU HUANG ${ }^{1}$, JING CAI $^{1}$ and ZEHUA WANG ${ }^{1}$ \\ ${ }^{1}$ Department of Obstetrics and Gynecology, Union Hospital, Tongji Medical College, Huazhong University of Science and \\ Technology; ${ }^{2}$ Department of Obstetrics and Gynecology, Tongren Hospital of Wuhan University (Wuhan Third Hospital), \\ Wuhan, Hubei 430022, P.R. China; ${ }^{3}$ University Clinic for Medical Radiation Physics, Medical Campus Pius-Hospital, \\ Carl von Ossietzky University, Oldenburg D-26121, Germany
}

Received October 24, 2017; Accepted July 13, 2018

DOI: $10.3892 / \mathrm{mmr} .2018 .9452$

\begin{abstract}
Dicer is an essential enzyme that processes micro (mi)-RNA precursors into mature miRNAs, and serves a critical role in cancer development and progression by regulating gene expression. However, the role of Dicer in cisplatin-mediated apoptosis and chemotherapy resistance in epithelial ovarian cancer (EOC) cells is poorly understood. In the present study, Dicer was expressed at low levels in cisplatin-resistant A2780 cells when compared with parental cells. In addition, knocking down Dicer using short hairpin RNA decreased the sensitivity of A2780 and CAOV3 cells to cisplatin. Furthermore, downregulating Dicer significantly inhibited cisplatin-induced apoptosis in ovarian cancer cells, and decreased the levels of proteins involved in apoptosis signaling pathways, including P73, P63, P53, caspase-9 and caspase-3. These findings indicated that Dicer may be a promising target for overcoming drug resistance in ovarian cancer.
\end{abstract}

\section{Introduction}

Ovarian cancer is the most lethal malignant gynecologic cancer (1). More than $70 \%$ of ovarian cancer patients are diagnosed at an advanced stage due to a lack of early-stage symptoms, and the overall 5-year survival rates are less than $30 \%$ (2). Even after receiving primary cytoreductive surgery and platinum-based chemotherapy, 60-70\% of EOC patients eventually undergo relapse, which is largely due to

Correspondence to: Dr Zehua Wang, Department of Obstetrics and Gynecology, Union Hospital, Tongji Medical College, Huazhong University of Science and Technology, 1277 Jiefang Avenue, Wuhan, Hubei 430022, P.R. China

E-mail: zehuawang@163.net

*Contributed equally

Key words: Dicer, ovarian cancer, cisplatin, chemoresistance chemotherapy resistance. Unfortunately, the specific mechanism of chemoresistance remains unclear.

Dicer belongs to the RNase III family of enzymes whose function is central to the processing of miRNA precursors into mature miRNAs in the cytoplasm (3). Dicer directly affects the levels and activity of miRNAs (4). Numerous recent studies have revealed the importance of Dicer in cancer development, chemoresistance and progression, but its expression levels are not consistent in different types of tumors. There are tissue-specific effects associated with the aberrant expression of Dicer (5). Recent research has shown that Dicer is associated with chemoresistance in colon cancer cells (6), breast cancer (7) and lung cancer cells (8). In ovarian cancer, it was reported that the deletion of Dicer could promote epithelial ovarian cancer (EOC) progression by increasing PDIA3 expression levels (9). In addition, several studies indicated that low levels of Dicer expression were significantly associated with high-grade histologic features and poor responses to chemotherapy $(10,11)$. Although these findings support the hypothesis that Dicer plays an essential role in ovarian cancer chemoresistance, the underlying molecular mechanism is unclear.

Therefore, in the present study, we aimed to demonstrate whether downregulating Dicer can mediate cell apoptosis and regulate cisplatin resistance in ovarian cancer cells in vitro.

\section{Materials and methods}

Cell lines and culture conditions. The human EOC cell lines A2780 and CAOV3 were purchased from the China Center for Type Culture Collection (CCTCC, Wuhan University, China). A cisplatin-resistant subline (A2780/DDP) was generated and maintained by our laboratory (12). Cisplatin was purchased from Shandong Qilu Pharmaceutical Factory. All cell lines were cultured in RPMI-1640 medium (Gibco; Thermo Fisher Scientific, Inc., Waltham, MA, USA) with $10 \%$ fetal calf serum (FCS; Gibco; Thermo Fisher Scientific, Inc.). Cells were maintained in a $37^{\circ} \mathrm{C}$ humidified incubator with an atmosphere of $5 \% \mathrm{CO}_{2}$. A2780/DDP cells were cultured without cisplatin for more than 1 month prior to use in this study to exclude stress reactions mediated by drug treatment. 
Dicer shRNA stable transfection. The Dicer-shRNA and control-shRNA vectors were purchased from Shanghai GenePharma (Shanghai, China). The target sequence for Dicer-shRNA was 5'-GCCAAGGAAATCAGCTAAATT-3'. A2780 and CAOV3 cells were seeded in a six-well plate at a concentration of $5 \times 10^{5}$ cells per well. Transfection was performed via lentivirus infection according to the manufacturer's instructions. Then, the cells were selected by $2 \mu \mathrm{g} / \mathrm{ml}$ puromycin until resistant cell colonies were obtained. The stably transfected cells were harvested $48 \mathrm{~h}$ after transfection and used for further assays. To verify the knockdown efficiency, mRNA and protein levels in the stably transfected cells were analyzed by reverse transcription-quantitative polymerase chain reaction (RT-qPCR) and western blot assays.

$R T-q P C R$. Total RNA was extracted from the cell lines using TRIzol reagent (Invitrogen; Thermo Fisher Scientific, Inc.), and cDNA was synthesized using an RT kit (Toyobo Life Science, Osaka, Japan) according to the manufacturer's protocol. Dicer and $\beta$-actin were measured by a SYBR Green (Takara Bio, Inc., Otsu, Japan) qPCR assay. The sequences of the Dicer primers were as follows: Upstream, 5'-GTGGTTCGTTTT GATTTGCCC-3' and downstream, 5'-CGTGTTGATTGT GACTCGTGGA-3' (NM_001195573.1). $\beta$-actin was used for normalization, and the primer sequences were as follows: Upstream, 5'-GCCAACACAGTGCTGTCTGG-3' and downstream, 5'-GCTCAGGAGGAGCAATGATCTTG-3'. Dicer was amplified under the following $\mathrm{qPCR}$ reaction conditions: Initial denaturation at $95^{\circ} \mathrm{C}$ for $1 \mathrm{~min}$, followed by 40 cycles of denaturation at $95^{\circ} \mathrm{C}$ for $15 \mathrm{sec}$, and annealing at $62^{\circ} \mathrm{C}$ for 1 min. All reactions were run on an Applied Biosystems 7500 Real-time PCR system (Applied Biosystems; Thermo Fisher Scientific, Inc.). The $2^{-\Delta \Delta C q}$ method was used to calculate relative changes in gene expression (13).

Western blotting. Cellular proteins were lysed in NP40 Cell Lysis Buffer (Beyotime Institute of Biotechnology, Haimen, China) containing $1 \mathrm{mM}$ protease inhibitor cocktail (Sigma-Aldrich; Merck KGaA, Darmstadt, Germany) at $4^{\circ} \mathrm{C}$ for $30 \mathrm{~min}$. The protein concentrations were determined using the bicinchoninic acid (BCA) method. Fifty micrograms of total protein from each sample was resolved by $8 \%$ (Dicer) or 10\% (P53, P63, P73, caspase-9 and caspase-3) SDS-PAGE and then was transferred onto polyvinylidene difluoride (PVDF) membranes (Bio-Rad Laboratories, Inc., Hercules, CA, USA). Membranes were incubated with mouse anti-Dicer (1:500 dilution; Abcam, Cambridge, MA, USA), rabbit anti-P53 (1:1,000 dilution; Cell Signaling Technology, Inc., Danvers, MA, USA), rabbit anti-P63 (1:500 dilution; Abcam), rabbit anti-P73 (1:500 dilution; Abcam), rabbit anti-caspase-9 (1:1,000 dilution; Cell Signaling Technology, Inc.), rabbit anti-caspase-3 (1:2,000 dilution; Cell Signaling Technology, Inc.), rabbit anti-P21 (1:1,000 dilution; Cell Signaling Technology, Inc.) and mouse anti- $\beta$-actin (1:5,000 dilution; Wuhan Boster Biological Technology, Ltd., Wuhan, China). $\beta$-Actin was used as a loading control. The primary antibodies were detected using HRP-conjugated secondary antibody (1:5,000 dilution; Wuhan Boster Biological Technology, Ltd.). The final immunoblots were detected by using an enhanced chemiluminescence system (Pierce; Thermo Fisher Scientific, Inc.). Protein bands were quantitated after scanning using Quantity One software (Bio-Rad Laboratories, Inc.).

MTT assays. Chemoresistance to cisplatin was assessed using 3-(4,5-dimethylthiazol-2-yl)-2,5-diphenyltetrazolium bromide (MTT) assays. Briefly, cells were plated in triplicate in a 96-well plate at a density of 5000 cells/well. Cells were treated with cisplatin at various concentrations ranging from 2.5 to $100 \mu \mathrm{M}$ for an additional $48 \mathrm{~h}$. After that, $20 \mu \mathrm{l}$ of MTT (Sigma-Aldrich; Merck KGaA) at a final concentration of $0.5 \mathrm{mg} / \mathrm{ml}$ was added to each well and incubated at $37^{\circ} \mathrm{C}$ for $6 \mathrm{~h}$. Then, the cells were dissolved in $100 \mu \mathrm{l}$ of DMSO by incubating overnight at $37^{\circ} \mathrm{C}$. The absorbance at a wavelength of $570 \mathrm{~nm}$ was measured using an iMark microplate reader (serial no. 10601; Bio-Rad Laboratories, Inc.). The percentage of cell survival at each dose was calculated as the absorbance ratio of treated to untreated cells. The 50\% inhibitory concentration $\left(\mathrm{IC}_{50}\right)$ values were calculated by linear interpolation. The data shown are representative of three independent experiments.

Hoechst 33342 staining. Cells were plated in triplicate in a six-well plate at a density of $1 \times 10^{5}$ cells/well and incubated for $24 \mathrm{~h}$. After that, A2780 cells were treated with $40 \mu \mathrm{M}$ cisplatin, and CAOV3 cells were treated with $30 \mu \mathrm{M}$ cisplatin. After a $48 \mathrm{~h}$ incubation at $37^{\circ} \mathrm{C}$, the cells were fixed in $4 \%$ paraformaldehyde for $10 \mathrm{~min}$ at room temperature. Finally, $1 \mu \mathrm{g} / \mathrm{ml}$ Hoechst 33342 (Invitrogen; Thermo Fisher Scientific, Inc.) was added for $5 \mathrm{~min}$. Then, images of the six-well plates mounted with emission fluorescence were acquired with a fluorescence microscope (model IX70; Olympus Corporation, Tokyo, Japan) and Image-Pro Plus software (Media Cybernetics, Inc., Rockville, MD, USA). The Hoechst reagent was taken up by nuclei, and the nuclei of apoptotic cells exhibited bright blue fluorescence. Each experiment was repeated separately three times.

Flow cytometric assays. Cells were plated in triplicate in a six-well plate at a density of $1 \times 10^{5}$ cells/well and incubated for $24 \mathrm{~h}$. After that, A2780 cells were treated with $40 \mu \mathrm{M}$ cisplatin, and CAOV3 cells were treated with $30 \mu \mathrm{M}$ cisplatin. After a $48 \mathrm{~h}$ incubation at $37^{\circ} \mathrm{C}$, the cells were harvested, trypsinized and washed with cold PBS. Subsequently, the cells were stained with APC Annexin V and propidium iodide (cat. no. 640932; BioLegend, Inc., San Diego, CA, USA). The stained cells were immediately analyzed by an LSR flow cytometer (BD Biosciences, Franklin Lakes, NJ, USA), and the data were analyzed using ModFit LT software (Verity Software House, Inc., Topsham, ME, USA).

Statistical analysis. Data are presented as the mean \pm standard deviation $(n \geq 3)$. Statistical comparisons between groups were estimated by two-tailed Student's t-tests or one-way analysis of variance followed by Dunnett's post hoc test. Data analyses were carried out using the SPSS v.13.0 statistical software package (SPSS, Inc., Chicago, IL, USA). P<0.05 was considered to indicate a statistically significant difference.

\section{Results}

Dicer expression levels are decreased in cisplatin-resistant ovarian cancer cells. To investigate whether the expression 

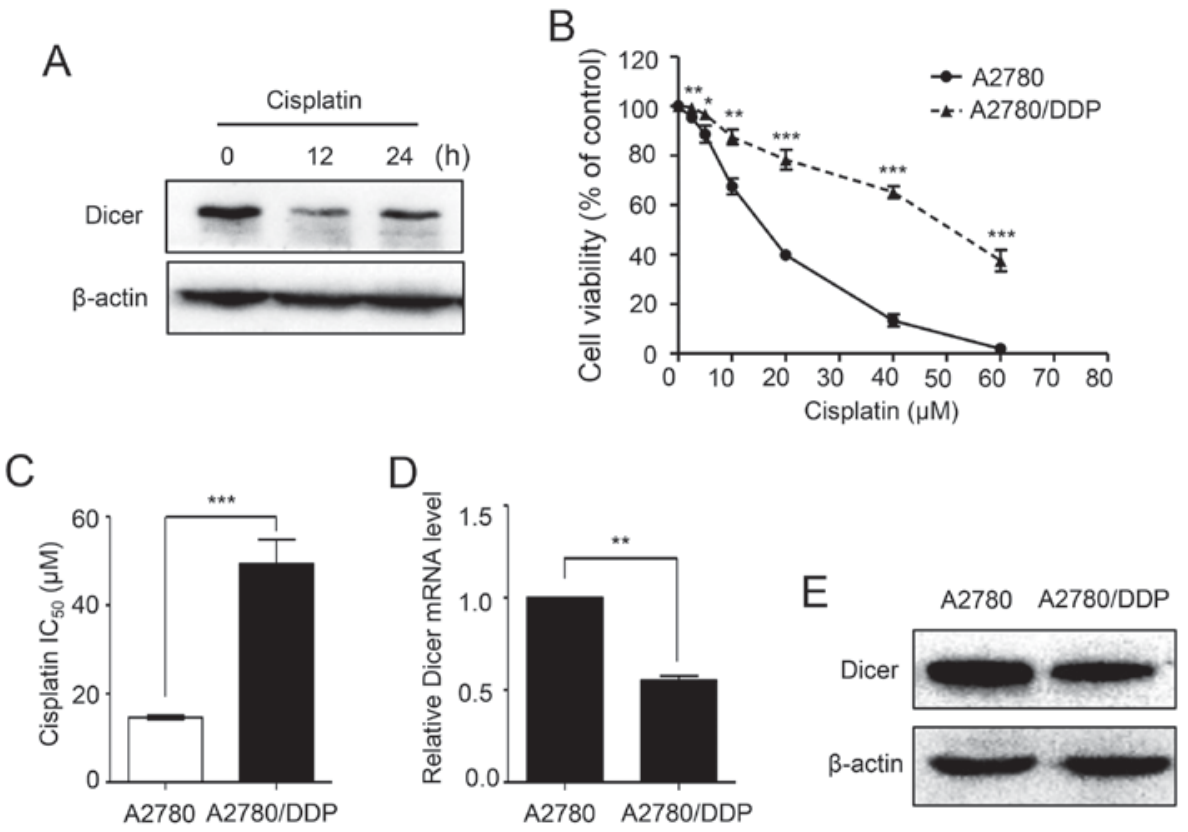

Figure 1. Dicer expression levels are decreased in A2780/DDP cells. (A) Western blots presenting the Dicer protein levels in A2780 cells following treatment with $20 \mu \mathrm{M}$ cisplatin for 0,12 and $24 \mathrm{~h}$. (B) Cell survival following cisplatin treatment was significantly higher in A2780/DDP cells when compared with A2780 cells, as determined by MTT assays. (C) The cisplatin $\mathrm{IC}_{50}$ was significantly increased in A2780/DDP cells when compared with that in A2780 cells following the MTT assays. (D) The relative level of Dicer mRNA was decreased in A2780/DDP cells when compared with A2780 cells, as determined by reverse transcription-quantitative polymerase chain reaction. Dicer expression was normalized to $\beta$-actin. (E) Dicer protein expression was downregulated in A2780/DDP cells when compared with A2780 cells according to western blot analyses. ${ }^{*} \mathrm{P}<0.05,{ }^{* * *} \mathrm{P}<0.01$ and ${ }^{* * * *} \mathrm{P}<0.001$, vs. A2780/as indicated.

of Dicer is affected by cisplatin, we treated A2780 cells with $20 \mu \mathrm{M}$ cisplatin for different periods of time. Western blot analyses showed that the protein expression levels of Dicer were dramatically decreased after cisplatin treatment for 12 and $24 \mathrm{~h}$ (Fig. 1A). To identify the role of Dicer in cisplatin resistance, first, the cisplatin sensitivities of parental A2780 cells and A2780/DDP were assessed using MTT assays. As demonstrated in Fig. 1B, the A2780/DDP cells dose-survival curve was shifted to the right, and the cells became more resistant to cisplatin. The cisplatin $\mathrm{IC}_{50}$ value for the A2780/DDP cells was 3.38-fold higher than that of A2780 cells (49.31 vs. $14.61 \mu \mathrm{M} ; \mathrm{P}<0.001$; Fig. 1C). Then, the expression of Dicer in A2780 and A2780/DDP cells was analyzed by RT-qPCR and western blot assays. The results showed that Dicer mRNA and protein levels were significantly lower in A2780/DDP cells than in parental A2780 cells (Fig. 1D and E).

Dicer knockdown increases cisplatin resistance in ovarian cancer cells. To study the effects of Dicer knockdown on cisplatin sensitivity, first, A2780 and CAOV3 cells were transfected with a shRNA plasmid against Dicer (shDicer) or a scrambled control plasmid (shNC). Compared with those in the shNC cells, Dicer mRNA levels were decreased by 77.3 and $73.09 \%$ in A2780/shDicer and CAOV3/shDicer cells, respectively ( $\mathrm{P}<0.01$; Fig. 2A). Similarly, western blot analyses confirmed Dicer knockdown after shDicer transfection (Fig. 2B). Then, the sensitivities of parental control, shNC and shDicer cells to cisplatin were assessed by MTT assays. The results showed that the dose-survival curves in the A2780/shDicer and CAOV3/shDicer cells were shifted to the right, and the cells became more resistant to cisplatin than the shNC cells (Fig. 2C and D). In addition, compared with those of the corresponding shNC cells, the $\mathrm{IC}_{50}$ values for cisplatin were increased by 2.37- and 1.68-fold in the A2780/shDicer (14.42 vs. $34.2 \mu \mathrm{M} ; \mathrm{P}<0.001$, Fig. $2 \mathrm{D}$ ) and $\mathrm{CAOV} 3 / \mathrm{shDicer}$ cells (20.34 vs. $34.2 \mu \mathrm{M} ; \mathrm{P}<0.001$, Fig. 3D), respectively.

Dicer knockdown inhibits cisplatin-induced cell apoptosis in ovariancancercells. Cisplatin is primarily believed to kill caner cells by inducing cell apoptosis. To explore the effects of Dicer on cisplatin-induced cancer cell apoptosis, A2780 and CAOV3 cells were treated with cisplatin for $48 \mathrm{~h}$ at concentrations of 40 and $30 \mu \mathrm{M}$, respectively, and Hoechst 33342 staining was then performed. According to fluorescence microscopy images, the shDicer cells had significantly less nuclear fragmentation than the parental control cells and the shNC cells in both A2780 $((5.22 \pm 0.41),(13.14 \pm 0.84)$ and $(13.33 \pm 0.69) \%$ apoptosis rates for shDicer, parental control and shNC cells, respectively, $\mathrm{P}<0.001)$ and CAOV3 cells $((5.14 \pm 0.46),(12.17 \pm 0.79)$ and $(12.39 \pm 0.73) \%$ apoptosis rates for shDicer, parental control and shNC cells, respectively, $\mathrm{P}<0.001$; Fig. $3 \mathrm{~A}$ and B). Consistently, Annexin V/propidium iodide dual staining followed by flow cytometric analyses showed that compared with the shNC cells, the proportion of apoptotic shDicer cells was decreased by 1.88-, 3.11- and 6.95-fold in A2780 cells after treatment with cisplatin at concentrations of 0,20 and $40 \mu \mathrm{M}$, respectively (Fig. 3C and D); the proportion of apoptotic shDicer cells was decreased by 2.26-, 4.27- and 10.17-fold in CAOV3 cells after treatment with cisplatin at concentrations of 0,15 and $30 \mu \mathrm{M}$, respectively (Fig. 3C and E).

Dicer knockdown decreases apoptosis-related protein expression levels. To investigate whether Dicer affects the expression of apoptosis-related proteins in cancer cells 
A

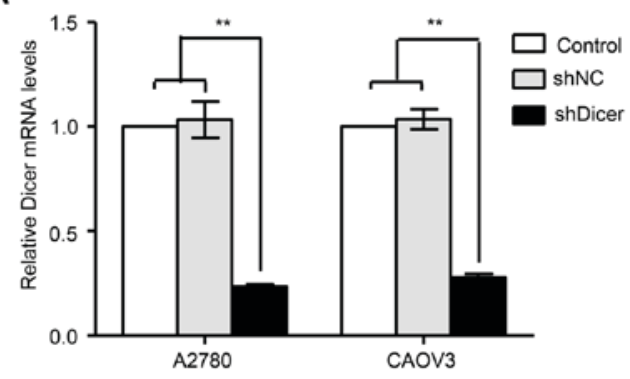

C

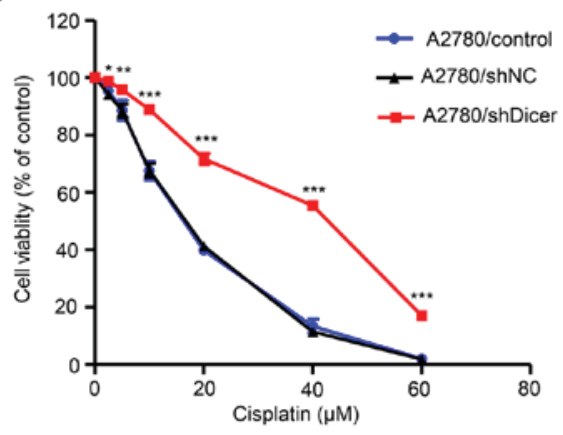

E

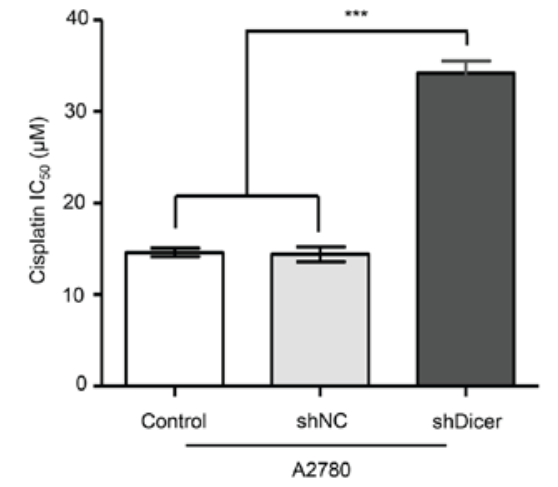

B

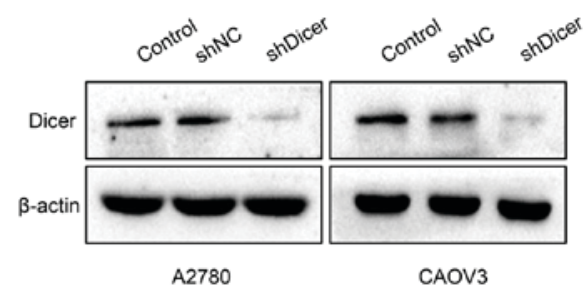

$\mathrm{D}$

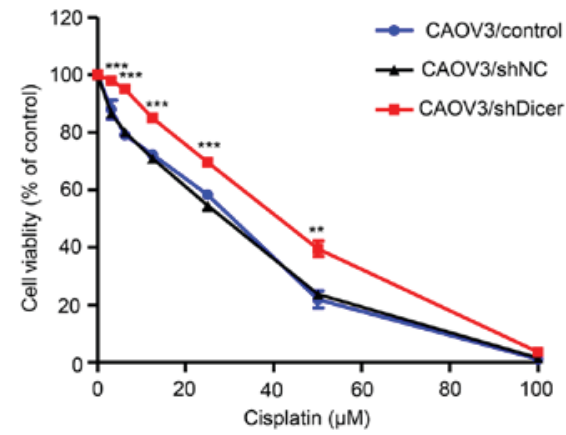

F

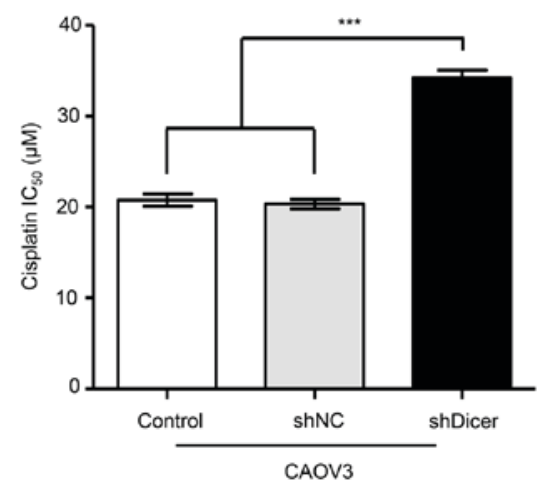

Figure 2. Dicer knockdown increases cisplatin resistance in ovarian cancer cells. (A) The expression levels of Dicer mRNA and (B) protein in A2780 and CAOV3 cells treated with Dicer-targeted shRNA (shDicer) or scrambled shRNA (shNC) and in parental control cells was determined by reverse transcription-quantitative polymerase chain reaction and western blot analyses, respectively. ${ }^{* *} \mathrm{P}<0.01$, as indicated. The relative viability of $(\mathrm{C}) \mathrm{A} 2780 / \mathrm{shDicer}$ vs. A2780/shNC and that of (D) CAOV3/shDicer vs. CAOV3/shNC were measured by MTT assays after treating cells with the indicated concentrations of cisplatin. (E and F) The $\mathrm{IC}_{50}$ for cisplatin in the ovarian cancer cell lines, (E) A2780 and (F) CAOV3 are shown. ${ }^{* * *} \mathrm{P}<0.001$, as indicated. $\mathrm{IC}_{50}$, half-maximal inhibitory concentration; shRNA, short hairpin RNA; NC, negative control.

exposed to cisplatin, P73, P63, P53, cleaved caspase-9, cleaved caspase-3 levels and P21 were measured in ovarian cancer cells that were treated with cisplatin for $48 \mathrm{~h}$. The treatment concentrations of cisplatin were 0,20 and $40 \mu \mathrm{M}$ for A2780 cells and 0,15 and $30 \mu \mathrm{M}$ for CAOV3 cells. Western blot analyses showed that the cisplatin-mediated increases in the expression levels of P73, P63, P53, P21, cleaved caspase-9 and cleaved caspase-3 were concentration-dependent in both shNC and shDicer cells; however, these expression levels were generally lower in the shDicer cells, especially the active fragments of caspase-9 and caspase-3 (Fig. 4).

\section{Discussion}

In the present study, we showed that decreased Dicer expression levels contributed to cisplatin resistance in ovarian cancer cells. Our results indicate that Dicer knockdown could protect ovarian cancer cells from cisplatin-induced apoptosis by decreasing the expression levels of proteins involved in apoptosis signaling pathways, including P73, P63, P53, caspase-9 and caspase- 3 . These findings provide novel insight into the mechanism of chemoresistance in ovarian cancer.

In our study, we found a dramatic decrease in Dicer protein level $1 \mathrm{~h}$ after cisplatin treatment. Previous study reported that cisplatin-induced proteasome degradation cause rapid down-regulation of hCTR1 (human copper transporter 1) expression in human ovarian carcinoma cells (14). In addition, another research indicated that cisplatin could induce EGFR phosphorylation and subsequent ubiquitination and degradation in head and neck cancer (15). Whether the instant downregulation of Dicer after exposure to cisplatin is caused by cisplatin-induced protein degradation or not need to be further explored. 
A

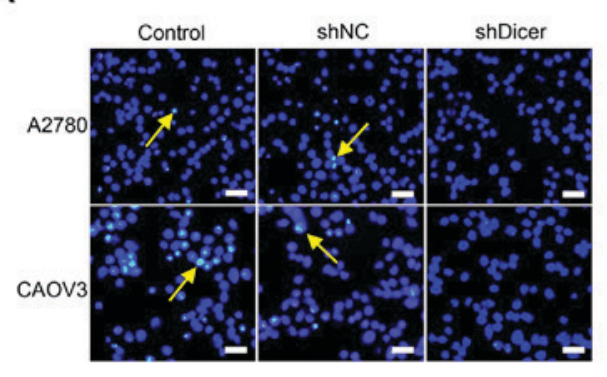

B

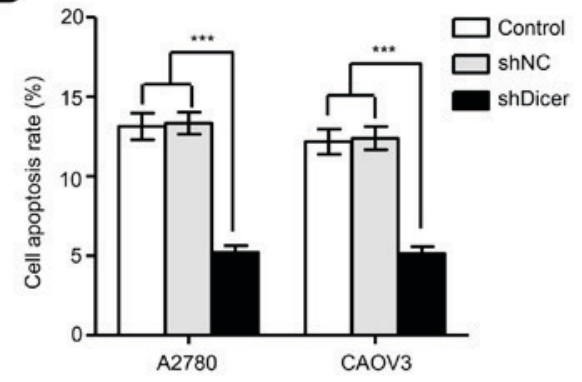

C

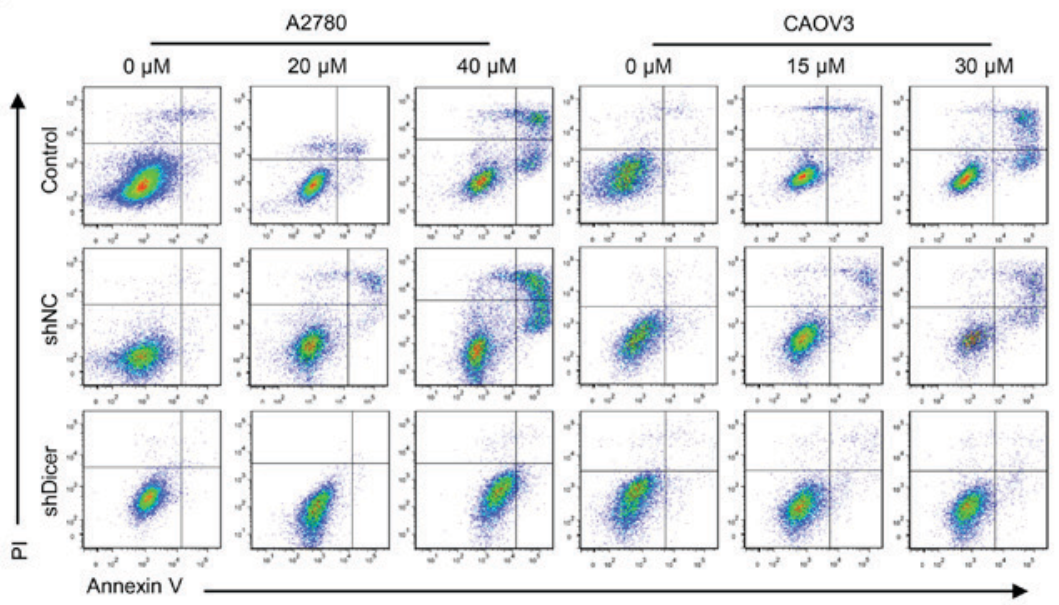

D

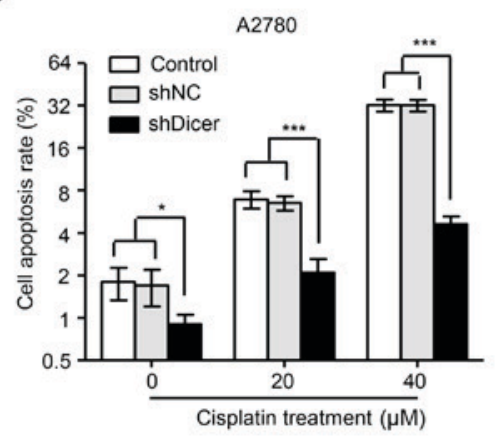

E

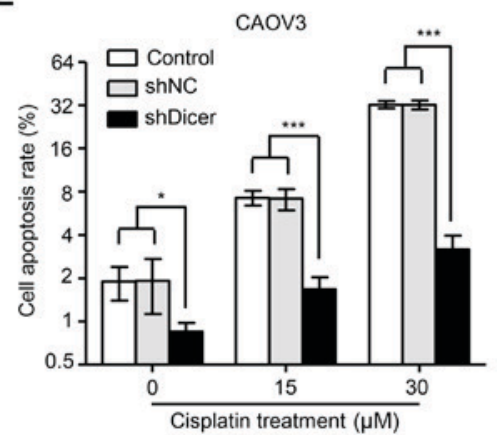

Figure 3. Dicer knockdown inhibits cisplatin-induced cell apoptosis in ovarian cancer cells. (A) Representative fluorescence images of A2780 and CAOV3 parental control, shNC and shDicer cells stained with Hoechst 33342 (blue) following cisplatin treatment. The yellow arrows indicate apoptotic cells with nuclear fragmentation. Scale bars, $50 \mu \mathrm{m}$. (B) The percentages of apoptotic cells were quantified by immunofluorescence analyses. (C) Apoptotic parental control, shNC and shDicer cells of the A2780 and CAOV3 cell lines were assessed using flow cytometry with Annexin V and PI double staining following cisplatin treatment. (D and E) The percentages of apoptotic (D) A2780 and (E) CAOV3 cells were quantified by flow cytometric analyses. Data are presented as the mean \pm standard deviation. ${ }^{*} \mathrm{P}<0.05$ and ${ }^{* * *} \mathrm{P}<0.001$, as indicated. shRNA, short hairpin RNA; NC, negative control; PI, propidium iodide.

A previous study revealed that reduced expression levels of Dicer in ovarian cancer were associated with increased tumor cell proliferation, enhanced migration ability and increased cisplatin resistance (11). In our previous study, we found that downregulation of Dicer could promote proliferation, colony formation, and migration ability of A2780, CAOV3, and SKOV3 ovarian cancer cells. Besides, proteomic profiling assay showed that Dicer knockdown promoted ovarian cancer progression by elevating PDIA3 expression (9). Our study further revealed that the downregulation of Dicer could increase cisplatin resistance in ovarian cancer cells through inhibiting cisplatin-induced apoptosis and decreasing the levels of cleaved caspase-3 and caspase-9. Similarly, several recent reports have proven that decreased Dicer levels play an important role in drug resistance in different cancer types. In cervical cancer, a study showed that EZH2 inhibition could effectively increase the cisplatin sensitivity of HeLa/DDP cells by upregulating Dicer expression (16). In addition, the knockdown of Dicer resulted in resistance to 5-FU-based chemoradiotherapy and a poor prognosis in patients with oral squamous cell carcinoma (17). In addition, the upregulation of miR-18a decreased Dicer expression levels and conferred paclitaxel resistance in triple-negative breast cancer (7). These findings are consistent with our results. However, some studies have obtained the opposite results regarding the relationship between Dicer expression and drug resistance. In breast 


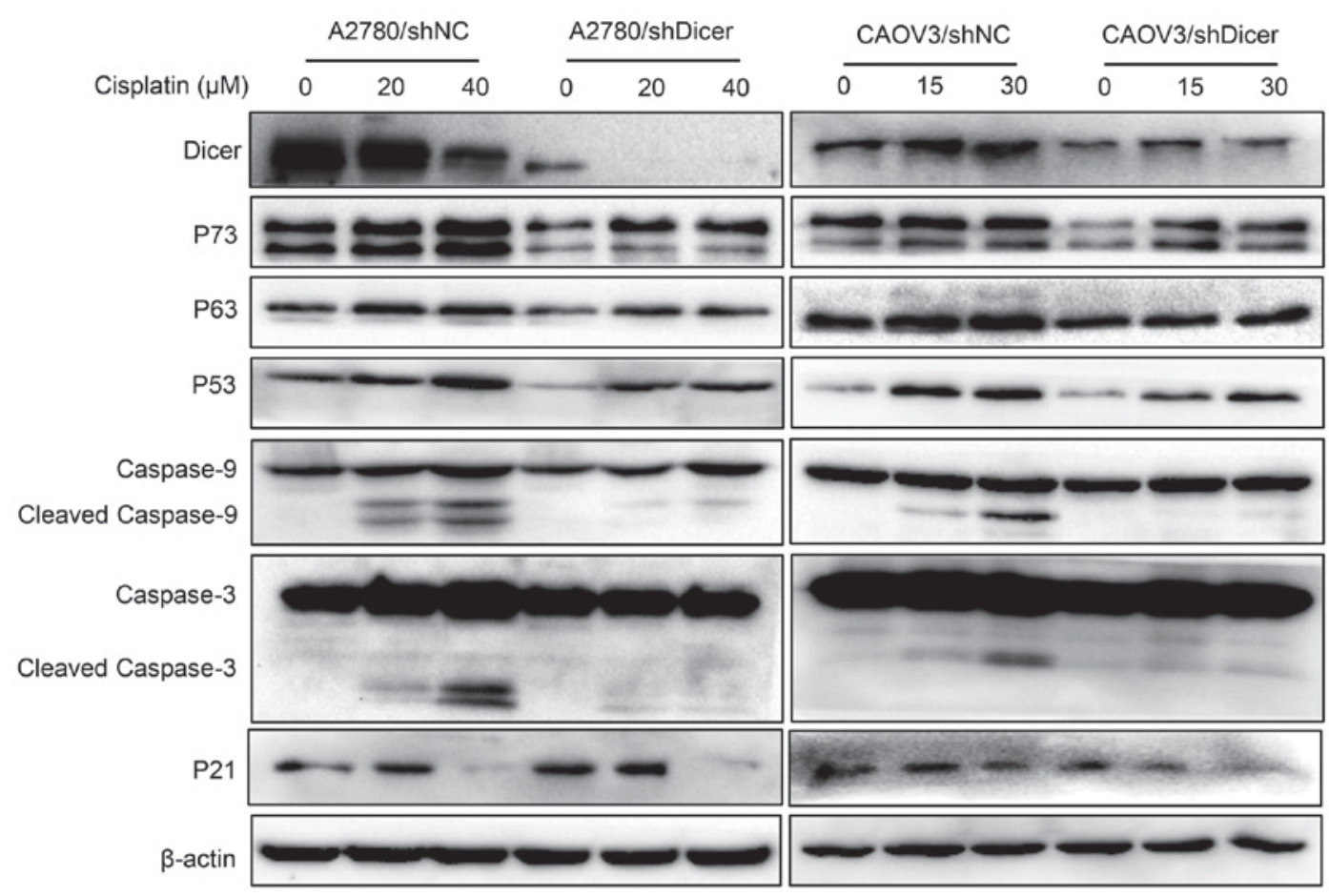

Figure 4. Dicer knockdown decreases the expression levels of apoptosis-associated proteins induced by cisplatin in ovarian cancer cells. Western blot analyses demonstrated that the expression levels of proteins involved in apoptosis signaling pathways, including P73, P63, P53, caspase-9 and caspase-3, were decreased in shDicer cells when compared with shNC cells following cisplatin treatment in the A2780 and CAOV3 cell lines. Western blot analyses also demonstrated that P21 protein expression was not affected by Dicer downregulation in A2780 and CAOV3 cells, while it was decreased following cisplatin treatment. The results were normalized to the expression of the reference protein $\beta$-actin. shRNA, short hairpin RNA; NC, negative control.

cancer, $\mathrm{Bu}$ et al reported that the suppression of Dicer could result in G1 arrest in MCF-7 cells and increase their sensitivity to cisplatin (18). It has also been reported that downregulating Dicer could increase gefitinib sensitivity in human lung cancer cells (8). These opposite results may be attributed to the complicated structure and function of Dicer.

Previous studies have demonstrated that Dicer might promote cell apoptosis through regulating apoptosis-related genes $(19,20)$. It is well-known that P53 superfamily proteins including P53, P63 and P73 function in mediating apoptosis in the presence of many stress stimuli, including activation of oncogenes and DNA damage $(21,22)$. As is known, p53 plays a central role in tumor suppression, while which is the most frequently mutated gene in human cancer, and over half of human cancers contain p53 mutations. Although the critical role of wild-type p53 in tumor suppression has been firmly established, mounting evidence has demonstrated that many tumor-associated mutant p53 proteins not only lose the tumor-suppressive function of wild-type p53 but also gain new activities to promote tumorigenesis independently of wild-type p53, termed gain-of-function. Mutant p53 protein often accumulates to very high levels in tumors, contributing to malignant progression (21). In addition, caspase- 9 and caspase- 3 can be activated in response to cisplatin-induced apoptosis (23). Our findings indicate that the expression levels of proteins involved in apoptosis signaling pathways, including P73, P63, P53, caspase- 9 and caspase- 3 , were decreased in ovarian cancer cells after Dicer knockdown. Therefore, our results indicate that Dicer might play a specific role in cisplatin-induced apoptosis in ovarian cancer cells. Besides, we found that the p21 protein expression was not affected by Dicer down-regulation in A2780 and CAOV3 cells, while it was decreased under cisplatin treatment. The cisplatin induced reduction of P21 was similar in the A2780 cells with or without Dicer depletion, but more intensive in the CAOV3 cells with Dicer depletion compared with control, which may be due to the presence of P53 mutations in CAOV3, but not in A2780 (24). Recently, it has been reported that tumor-associated mutant $\mathrm{p} 53$ promoted cancer cell survival upon glutamine deprivation through p21 induction (25), which is consistent with our results. As is known, Dicer plays an important role in the maturation of miRNAs. Emerging evidence has indicated that low levels of some miRNAs are associated with drug resistance in cancer cells. For example, low miRNA-107 and low miRNA-204 were linked to chemo-resistance in non-small cell lung cancers and prostate cancers, respectively, because they target the mRNAs of antiapoptotic factors Bcl-w and zinc-finger E-box-binding homeobox 1 (ZEB1) $(26,27)$. These findings suggest the potential roles of microRNAs in regulating Dicer-mediated chemotherapy responses in tumor cells. However, whether the role of Dicer in cisplatin resistance in ovarian cancer is mediated by corresponding microRNAs needs to be further studied.

In summary, we found that low expression levels of Dicer can inhibit cisplatin-induced apoptosis in ovarian cancer cells, which in turn resulted in resistance to cisplatin. However, the present study also has several limitations. First, Dicer upregulation in the cisplatin resistant ovarian cancer cells was not performed because of technical difficulty. Second, only one cisplatin-resistant cell line was used. Third, the mechanisms through which Dicer regulates cell apoptosis remain unclear. Given that Dicer functions as an enzyme that catalyzes miRNA maturation and that several miRNAs have been identified as regulators of cell apoptosis, 
multiple miRNAs might be involved in Dicer-mediated cisplatin resistance in ovarian cancer. Moreover, our results suggest that the upregulation of Dicer might be a promising strategy for treating recurrent, cisplatin-resistant EOC.

\section{Acknowledgements}

Not applicable.

\section{Funding}

The present study was supported by the Natural Science Foundation of Hubei Province (grant no. 2014CFB996) and the Health and Family Planning Commission of Wuhan Municipality (grant no. WX17C10).

\section{Availability of data and materials}

All data generated or analyzed during the present study are included in this published article.

\section{Authors' contributions}

ZW and HC designed the present study. ZW and JC supervised the project. XW worked out almost all of the technical details and performed the functional experiments. QH and YW performed the experiments regarding molecular mechanisms. $\mathrm{ZH}$ provided technical support. XY, PJ and HC analyzed and interpreted the data. YW and JC collected the data, and drafted the manuscript in consultation with PJ and ZW. JC revised the manuscript substantively. All authors contributed to producing the manuscript and approved the final manuscript.

\section{Ethics approval and consent to participate}

Not applicable.

\section{Patient consent for publication}

Not applicable.

\section{Competing interests}

The authors declare that they have no competing interests.

\section{References}

1. Siegel R, Ma J, Zou Z and Jemal A: Cancer statistics, 2014. CA Cancer J Clin 64: 9-29, 2014.

2. Jayson GC, Kohn EC, Kitchener HC and Ledermann JA: Ovarian cancer. Lancet 384: 1376-1388, 2014.

3. Bernstein E, Caudy AA, Hammond SM and Hannon GJ: Role for a bidentate ribonuclease in the initiation step of RNA interference. Nature 409: 363-366, 2001.

4. Krol J, Loedige I and Filipowicz W: The widespread regulation of microRNA biogenesis, function and decay. Nat Rev Genet 11 597-610, 2010

5. Bahubeshi A, Tischkowitz $M$ and Foulkes WD: miRNA processing and human cancer: DICER 1 cuts the mustard. Sci Transl Med 3: 111ps46, 2011.

6. Chen X, Li WF, Wu X, Zhang HC, Chen L, Zhang PY, Liu LY, Ma D, Chen T, Zhou L, et al: Dicer regulates non-homologous end joining and is associated with chemosensitivity in colon cancer patients. Carcinogenesis 38: 873-882, 2017.
7. Sha LY, Zhang Y, Wang W, Sui X, Liu SK, Wang T and Zhang H: MiR-18a upregulation decreases Dicer expression and confers paclitaxel resistance in triple negative breast cancer. Eur Rev Med Pharmacol Sci 20: 2201-2208, 2016.

8. Chen JC, Su YH, Chiu CF, Chang YW, Yu YH, Tseng CF, Chen HA and Su JL: Suppression of Dicer increases sensitivity to gefitinib in human lung cancer cells. Ann Surg Oncol 21 (Suppl 4): S555-S563, 2014

9. Zhu Y, Cai L, Guo J, Chen N, Yi X, Zhao Y, Cai J and Wang Z: Depletion of Dicer promotes epithelial ovarian cancer progression by elevating PDIA3 expression. Tumour Biol 37: 14009-14023, 2016.

10. Merritt WM, Lin YG, Han LY, Kamat AA, Spannuth WA, Schmandt R, Urbauer D, Pennacchio LA, Cheng JF, Nick AM, et al: Dicer, Drosha, and outcomes in patients with ovarian cancer. N Engl J Med 359: 2641-2650, 2008.

11. Kuang Y, Cai J, Li D, Han Q, Cao J and Wang Z: Repression of Dicer is associated with invasive phenotype and chemoresistance in ovarian cancer. Oncol Lett 5: 1149-1154, 2013.

12. Hu S, Yu L, Li Z, Shen Y, Wang J, Cai J, Xiao L and Wang Z Overexpression of EZH2 contributes to acquired cisplatin resistance in ovarian cancer cells in vitro and in vivo. Cancer Biol Ther 10: 788-795, 2010.

13. Livak KJ and Schmittgen TD: Analysis of relative gene expression data using real-time quantitative PCR and the 2(-Delta Delta C(T)) method. Methods 25: 402-408, 2001.

14. Holzer AK and Howell SB: The internalization and degradation of human copper transporter 1 following cisplatin exposure. Cancer Res 66: 10944-10952, 2006.

15. Ahsan A, Hiniker SM, Ramanand SG, Nyati S, Hegde A, Helman A, Menawat R, Bhojani MS, Lawrence TS and Nyati MK: Role of epidermal growth factor receptor degradation in cisplatin-induced cytotoxicity in head and neck cancer. Cancer Res 70: 2862-2869, 2010.

16. Cai L, Wang Z and Liu D: Interference with endogenous EZH2 reverses the chemotherapy drug resistance in cervical cancer cells partly by up-regulating Dicer expression. Tumour Biol 37: 6359-6369, 2016.

17. Kawahara K, Nakayama H, Nagata M, Yoshida R, Hirosue A Tanaka T, Nakagawa Y, Matsuoka Y, Kojima T, Takamune Y, et al: A low Dicer expression is associated with resistance to 5-FU-based chemoradiotherapy and a shorter overall survival in patients with oral squamous cell carcinoma. J Oral Pathol Med 43: 350-356, 2014.

18. Bu Y, Lu C, Bian C, Wang J, Li J, Zhang B, Li Z, Brewer G and Zhao RC: Knockdown of Dicer in MCF-7 human breast carcinoma cells results in G1 arrest and increased sensitivity to cisplatin. Oncol Rep 21: 13-17, 2009.

19. Nakagawa A, Shi Y, Kage-Nakadai E, Mitani S and Xue D: Caspase-dependent conversion of Dicer ribonuclease into a death-promoting deoxyribonuclease. Science 328: 327-334, 2010.

20. Swahari V, Nakamura A, Baran-Gale J, Garcia I, Crowther AJ, Sons R, Gershon TR, Hammond S, Sethupathy P and Deshmukh M: Essential function of dicer in resolving DNA damage in the rapidly dividing cells of the developing and malignant cerebellum. Cell Rep 14: 216-224, 2016.

21. Aubrey BJ, Kelly GL, Janic A, Herold MJ and Strasser A: How does p53 induce apoptosis and how does this relate to p53-mediated tumour suppression? Cell Death Differ 25: 104-113, 2018.

22. Mollereau B and Ma D: The p53 control of apoptosis and proliferation: Lessons from Drosophila. Apoptosis 19: 1421-1429, 2014.

23. Gonzalez VM, Fuertes MA, Alonso C and Perez JM: Is cisplatin-induced cell death always produced by apoptosis? Mol Pharmacol 59: 657-663, 2001.

24. Domcke S, Sinha R, Levine DA, Sander C and Schultz N: Evaluating cell lines as tumour models by comparison of genomic profiles. Nat Commun 4: 2126, 2013

25. Tran TQ, Lowman XH, Reid MA, Mendez-Dorantes C, Pan M, Yang Y and Kong M: Tumor-associated mutant p53 promotes cancer cell survival upon glutamine deprivation through p21 induction. Oncogene 36: 1991-2001, 2017.

26. Lu C, Xie Z and Peng Q: MiRNA-107 enhances chemosensitivity to paclitaxel by targeting antiapoptotic factor Bcl-w in non small cell lung cancer. Am J Cancer Res 7: 1863-1873, 2017.

27. Wu G, Wang J, Chen G and Zhao X: microRNA-204 modulates chemosensitivity and apoptosis of prostate cancer cells by targeting zinc-finger E-box-binding homeobox 1 (ZEB1). Am J Transl Res 9: 3599-3610, 2017.

This work is licensed under a Creative Commons Attribution-NonCommercial-NoDerivatives 4.0 International (CC BY-NC-ND 4.0) License. 